$16^{\text {th }}$ International Congress of Metrology, 05008 (2013)

DOI: $10.1051 /$ metrology/201305008

(c) Owned by the authors, published by EDP Sciences, 2013

\title{
Application of TDCR-Geant4 modeling to activity standardization
}

\author{
Cheick Thiam ${ }^{\mathrm{a}}$, Christophe Bobin, Bruno Chauvenet and Jacques Bouchard \\ CEA, List, Laboratoire National Henri Becquerel (LNE-LNHB), F-91191 Gif-sur-Yvette Cedex, France
}

\begin{abstract}
The LNE-LNHB, in charge of the French standards for radionuclide metrology, develops primary measurement methods intended for the calibration of secondary measuring instruments, such as used in hospitals, to determine the activity of radiopharmaceuticals injected into patients. The triple-to-double coincidence ratio (TDCR) method, based on liquid scintillation counting, is widely implemented for radionuclides which are pure beta and electron capture emitters. This technique requires the mixing of the radioactive solution with a scintillator cocktail. When preparing samples, the existence of physicochemical processes such as chemi-luminescence can require to delay the measurements to achieve the necessary stability conditions, which is not always compatible for short halflife radionuclides. To avoid this constraint, a new approach using Cerenkov radiation as a method of light production is presented in this paper, applied to the standardization of the short-lived radiopharmaceutical ${ }^{11} \mathrm{C}\left(\mathrm{T}_{1 / 2} \sim 20 \mathrm{~min}\right)$. The method is based on comprehensive modeling of the TDCR counter (the experimental equipment) using a Geant 4 toolkit in order to calculate the detection efficiency.
\end{abstract}

\section{Introduction}

Among the medical applications using radioactive sources, PET-scan (Positron Emission Tomography) is an imaging technique performed to investigate the functioning of tissues and organs. This technique requires the injection of a small dose of the radioactive tracer into a vein and a gamma camera detects the radiation released by the tracer $\left(\right.$ e.g. $\left.{ }^{18} \mathrm{~F},{ }^{11} \mathrm{C}\right)$ to produce computer images. According to the organ or tissue studied and for radioprotection reasons, the activity injected, quantified in becquerel $(\mathrm{Bq})$, must be estimated with precision. The LNHB, in charge of the French standards for radionuclide metrology, develops primary measurement methods intended for the calibration of secondary measuring instruments used for example in hospitals. The triple-todouble-coincidence ratio (TDCR) method based on liquid scintillation (LS) counting is widely implemented for the measurements of radionuclides which decay by pure beta and electron capture emission. The TDCR method is based on a three-photomultiplier (PMT) detection system, and the activity is determined using the double- and triple-coincidences between PMTs. A statistical model of the light emission in the counter and the detection in the PMTs is required in order to calculate the detection efficiency [1]. Using the same experimental set-up, the method can be also applied to measurements of Cerenkov light when an additional free parameter is included into the statistical model to take into account the anisotropy of Cerenkov light emission [2]. As an alternative to this standard practice, a new approach based on the Geant4 Monte Carlo toolkit was recently developed at LNHB [3]. As an object-oriented and open-source set of libraries, the Geant4 code is able to simulate all processes related to the transport of charged particles and the ensuing generation of optical photons due to the scintillation process (radionuclide solution mixed with a scintillator cocktail) or the Cerenkov effect (radionuclide in an aqueous solution only). Based on complete modeling of the TDCR counter and all optical properties attached to the materials, the simulation allows optical photons to be tracked from their creation inside the LS-vial to the production of photoelectrons in the PMT, leading to the counting of double- and triple-coincidences.

In practice, as higher detection efficiencies are obtained with LS counting, this technique is widely used instead. However, in the case of radiopharmaceuticals, the short half-live imposes an additional constraint on the chemical preparation of the radioactive samples; the possibilities to optimize the chemical composition are therefore limited. This can be significant in LS counting as the radioactive solution is mixed with a scintillator cocktail. For instance, the photons due to the chemi-luminescence can interfere with those created by ionizing radiation; the subsequent instabilities can disturb the measurements. Cerenkov light radiation occurs when a charged particle moves through a dispersive medium faster than the group velocity of light in that medium. Cerenkov measurements represent then an interesting alternative to LS counting. Measurements can be carried out directly with aqueous solution without

\footnotetext{
${ }^{\mathrm{a}}$ Corresponding author: cheick.thiam@cea.fr
} 
the addition of a fluorescent cocktail. The quick and easy samples preparation can especially benefit radionuclides with a short half-life such as ${ }^{11} \mathrm{C}(\sim 20 \mathrm{~min})$.

Additionally, Cerenkov light emission is characterized by a threshold effect which can be used advantageously to discriminate potential impurities emitting radiation below the Cerenkov threshold. The beta-minus emitter ${ }^{32} \mathrm{P}$, having a maximum energy of $1.7 \mathrm{MeV}$, illustrates this situation well. Typical impurities are also beta-minus emitters, e.g. ${ }^{33} \mathrm{P}$ (maximum energy of $248 \mathrm{keV}$ ) and ${ }^{35} \mathrm{~S}$ (maximum energy of $167 \mathrm{keV}$ ). Energies lower than the Cerenkov threshold in the aqueous solution $(\sim 260 \mathrm{keV})$, are not detected.

This paper presents an application of the new TDCRGeant4 model for the standardization of ${ }^{11} \mathrm{C}$. The model was validated at first with the measurements of ${ }^{90} \mathrm{Y}$ (betaminus emitter with maximum energy of $2.3 \mathrm{MeV}$ ) using the Cerenkov radiation [4]. As Cerenkov emission is anisotropic and results from an electromagnetic process (no ionization quenching), these results provides a validation of the Geant4 model, without the need to simulate the physical chemistry processes causing liquid scintillation.

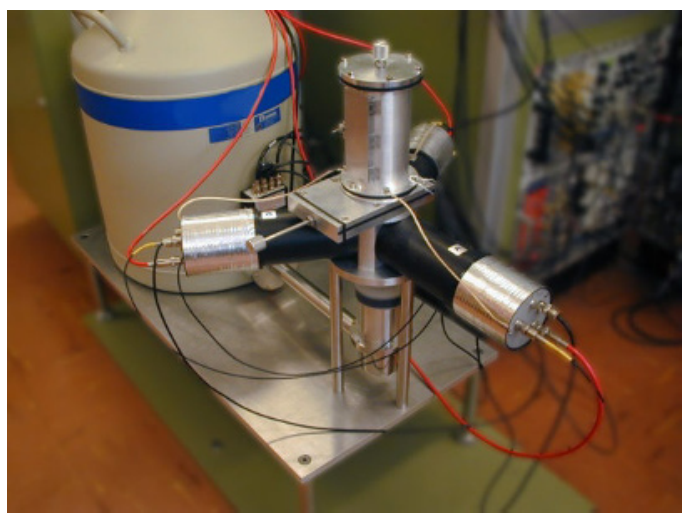

Figure 1. The three-photomultiplier counter used for the TDCR method at LNHB.

\section{Experimental setup}

The TDCR technique using LS counting is applied with a specific counter composed of three PMTs which are symmetrically placed around an optical chamber and, a vial containing the radioactive sample is placed within. A free parameter statistical model of the distribution of the scintillation photons and their detection probability in the counter is implemented to build a mathematical relation between the detection efficiency, corresponding to the double-coincidences and, the experimental TDCR value (triple-to-double coincidence ratio) which is used as an indicator of the detection efficiency. The detection efficiency is obtained by adjusting a free parameter representing both PMT-quantum efficiency and number of photons emitted per unit of energy released inside the LS cocktail. The TDCR counter (shown in Figure 1) is equipped with a XP2020Q photomultiplier model (by Photonis), with a fused-silica window and bialkali photocathode. Being sensitive to a large spectrum of wavelengths (160 to $650 \mathrm{~nm}$ ), this model is well adapted to detect the continuous spectral distribution of the Cerenkov light emission. The optical chamber is made of Teflon $^{\circledR}$ with a spherical reflecting cavity where the PMT-windows emerge around the LS-vial. The signals delivered by the PMTs are treated by a set of electronic modules, taking into account specific aspects such as the counting losses due to dead times [5].

\section{The TDCR counter modeling}

The TDCR counter was modeled using the Geant4 toolkit with a comprehensive description of the geometry (represented in Figure 2) and optical properties attached to the optical-chamber materials. The sample solution is defined as liquid water with a dispersive refractive index $(1.33$ at $400 \mathrm{~nm})$. The liquid-air and liquid-vial interfaces are described as dielectric-dielectric boundaries. The LSvial (a standard $20 \mathrm{~mL}$ borosilicate vial) is defined with dispersive refractive index (1.52 at $400 \mathrm{~nm})$ and a polished surface. The Teflon ${ }^{\circledR}$ reflecting cavity is defined as dielectric-metal using a lambertian surface type with a reflectivity equal to $95 \%$. To obtain realistic detection inside PMTs, some optical properties are also defined for the XP2020Q model. The fused-silica window is defined as a 52-mm-diameter cylinder with a spherical inner surface $(1.5 \mathrm{~mm}$ thick at the center, $7 \mathrm{~mm}$ at the fringe). The metallic ring (46 $\mathrm{mm}$ inner diameter) deposited around the bialkali coating for its voltage polarization is also described as a dielectric-metal boundary with the reflectivity equal to $95 \%$. A dispersive refractive index is attached to the fused-silica material $(1.47$ at $400 \mathrm{~nm})$ with a polished surface. The photocathode is simulated via its optical properties at the fused-silica inner boundary, to count the number of photons refracted between both surfaces. For a given application, the simulation runs according to the following steps:

- The initial particles are randomly generated in the whole volume corresponding to the radioactive sample in the LS-vial; energy release depends on the radionuclide decay scheme.

- The Cerenkov light is produced along the track of the charged particles inside the LS-vial and also the borosilicate wall.

- Each emitted photon is subsequently followed to account for the optical processes in the TDCR counter (refraction, reflection and absorption).

- The photoelectrons are produced in each PMT by applying a binomial trial (using the experimental quantum efficiency) on the photons refracted at the photocathode.

- The number of photoelectrons is obtained in the PMTs for each simulated event (corresponding to one disintegration). 
- The coincidences are calculated by using a second binomial trial representing the probability to reach the first dynode; at least one binomial success leads to a count in a PMT.

- The variation of detection efficiency as a function of experimental $T D C R$ value is subsequently obtained by adjusting this defocusing parameter.

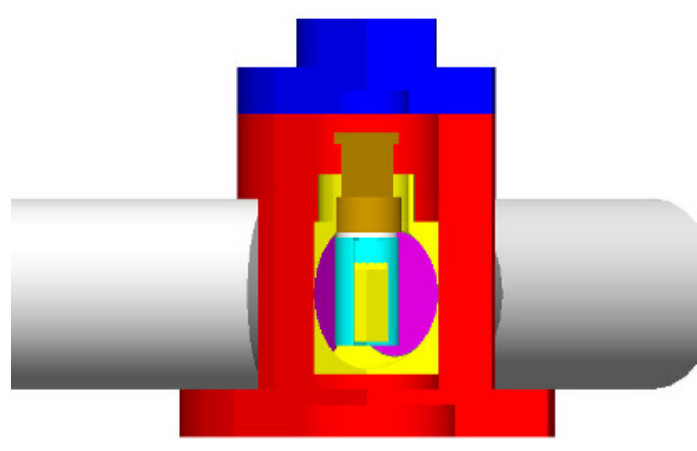

Figure 2. Geant 4 modeling of the TDCR counter including the counting vial developed for Cerenkov primary measurements.

All the simulations in this study were performed with the Geant4.9.4 release using the low-energy electromagnetic physics based on PENELOPE models.

\section{4. ${ }^{11} \mathrm{C}$ standardization}

For measurements, four samples were prepared in glass vials prefilled with $15 \mathrm{~mL}$ of inactive solution (10 $\mu \mathrm{g} / \mathrm{g}$ of carbon in $\mathrm{Na}_{2} \mathrm{CO}_{3}$ diluted within $\mathrm{H}_{2} \mathrm{O}$ ). The defocusing technique (based on voltage variation) applied to the photomultipliers gave experimental $T D C R$ values ranging from 0.25 to 0.3 . The corresponding detection efficiencies were calculated using the TDCR-Geant4 simulation. ${ }^{11} \mathrm{C}$ decays mainly by beta-plus emission with a maximum energy of $960 \mathrm{keV}$; the initial energies of positrons were randomly generated from a calculated spectrum. Figure 3 represents the activity concentration vs $T D C R$ value for three modeling configurations. To estimate a variability of the stochastic calculation, two modeling parameters having a significant impact on the photoelectrons counted inside each PMT were changed: on the one hand the distance of the PMT-window to the center of the optical cavity (16 and $17 \mathrm{~mm}$ ) and, on the other hand, the dispersive refractive index used for the bialkali-photocathode. For instance, the configuration Ha16 corresponds to the bialkali refractive index given by Harmer et al. [7] and a distance to the center equal to $16 \mathrm{~mm}$; the Mo17 configuration is based on the refractive index published by Motta and Schönert [8] and a position to the center equal to $17 \mathrm{~mm}$. The activity curve vs $T D C R$ value presents no systematic variation outside of the counting uncertainties; consequently, it can be considered that the simulation reproduces the experiment exactly.

The maximum double-coincidence detection efficiency equal to $\sim 23.5 \%$ is obtained for a $T D C R$ ratio of 0.305 . The corresponding focusing parameter is equal to 0.97 ; that is coherent with the maximum focusing parameter previously obtained for ${ }^{90} \mathrm{Y}$ (which gave a high detection efficiency of $\sim 70 \%$ ). The activity concentration was estimated equal to 410.0 (35) $\mathrm{MBq}^{-1} \mathrm{~g}^{-1}$ at the reference date, deduced from the mean value obtained with the configurations corresponding to the lowest and highest estimations (shown in Figure 3: Ha16 and Mo17). The associated uncertainty budget is reported in Table 1. To validate this result, another primary measurement based on the $4 \pi \beta-\gamma$ coincidence method was performed. The outcome activity concentration; 411 (2) $\mathrm{kBq} \cdot \mathrm{g}^{-1}$, is consistent with the result given by TDCR-Geant 4 .

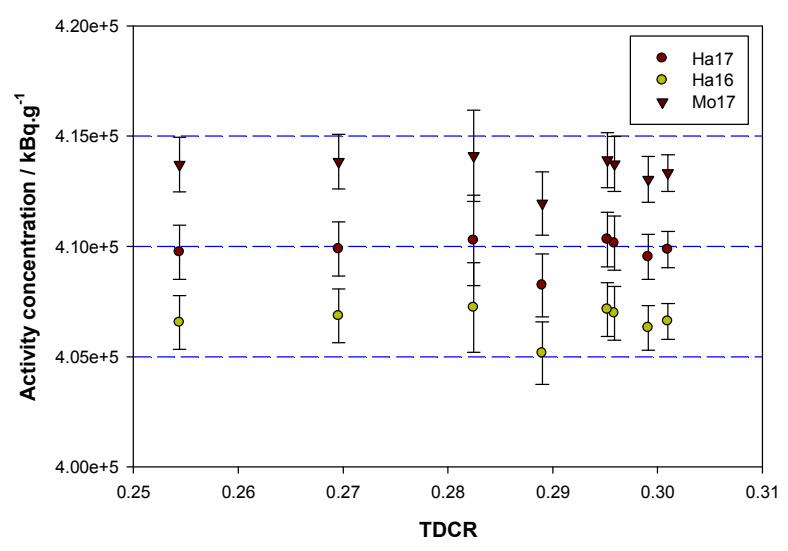

Figure 3. Activity concentration of ${ }^{11} \mathrm{C}$ vs $T D C R$ values. Three sets of points were obtained by varying the modeling parameters.

Table 1. Uncertainty budget associated to ${ }^{11} \mathrm{C}$ activity concentration obtained using TDCR-Geant 4 model.

\begin{tabular}{llc}
\hline $\begin{array}{l}\text { Uncertainty } \\
\text { component }\end{array}$ & Comment & $\begin{array}{c}\text { uncertainty } \\
\text { (\%) }\end{array}$ \\
\hline \hline statistics & standard deviation of 4 & 0.15 \\
& sources & \\
\hline background & & 0.05 \\
\hline live time & frequency clock at $1 \mathrm{MHz}$ & 0.1 \\
\hline weighing & pycnometer technique & 0.05 \\
\hline TDCR-Geant4 & variation of model & 0.8 \\
\hline PMT asymmetry & & 0.2 \\
\hline decay scheme & nuclear data & 0.1 \\
\hline \hline combined uncertainty & $\mathbf{0 . 8 5}$ \\
\hline
\end{tabular}

\section{Conclusion}

In practice, the accuracy in the activity measurement requires good preparation of the radioactive samples with special attention to the chemical stability. This constraint can be significant in the case of short-lived radionuclides such as ones used in nuclear medicine (less than 2 hours) and, in particular, when the measurements are carried out using liquid scintillation with the TDCR method. To overcome this, an alternative technique was suggested based on the Cerenkov radiation as the mode of light 
production in the radioactive solution. The measurements are done using the same detection system delivering double- and triple-coincidences between photomultipliers to estimate the activity of radioactive solution. The relation between the sample activity and the coincidence counting rates was performed through a stochastic model based the on Geant 4 code. The major interest of this stochastic approach is the ability to simulate refraction and reflection processes along the propagation of optical photons inside the detection system. In addition, the possibility to modify the simulation parameters is particularly interesting for the study of their influence on activity calculation. With a relative uncertainty lower than $1 \%$, this stochastic method gave satisfactory results for carbon-11 activity measurements. Already applied in the case of yttrium-90, the results show the capabilities of the modeling for the standardization of radionuclides over a large range of detection efficiency (between 20 and 70 $\%$ ). Since Cerenkov measurements are directly performed using aqueous solutions, this is an interesting alternative to liquid scintillation in the particular cases presenting chemical instability or in the presence of radionuclide impurities which can be discriminated due to the Cerenkov threshold effect.

As a consequence, the primary measurement method based on Cerenkov counting has been chosen and is being developed in the framework of the MetroMRT project (Metrology for Molecular Radiotherapy) of the European Metrology Research Program in order to establish a primary reference for yttrium- 90 microspheres used especially for liver cancer treatments. In practice, no primary reference standard is available; only the activity provided by the manufacturer is used to set a calibration factor for ionizing chambers used to estimate the delivered activity with an uncertainty of about $10 \%$.

Furthermore, several studies were carried out to extend the TDCR-Geant4 method to radionuclides in liquid scintillation cocktails, then including the liquid scintillation process. As for the classical model, the implementation uses the Birks expression to describe the nonlinearity of light emission due to ionization quenching. The TDCR-Geant4 model was applied to investigating the conditions of application of the statistical model used in the classical TDCR method for radionuclides with low-energy emission; in particular, to point out the problem of the stochastic dependence between photomultipliers [9].

\section{References}

1. R. Broda et al., "Radionuclide metrology using liquid scintillation counting", Metrologia, vol. 44, pp. 36-52, 2007.

2. K. Kossert, "Activity standardization by means of a new TDCR-Cerenkov counting technique", App. Radiat. and Isot., vol. 68, pp. 1116-1120, 2010.
3. S. Agostinelli et al., "Geant4 - a simulation toolkit", Nuclear Instruments and Methods, vol. 506, pp. 250-303, 2003.

4. C. Bobin et al., "Application of a stochastic TDCR model based on Geant4 for Cerenkov primary measurements", App. Radiat. and Isot., vol. 68, pp. 2366-2371, 2010.

5. J. Bouchard, P. Cassette, "MAC3: an electronic module for the processing of pulses delivered by a three photomultiplier LS counting system. Appl. Radiat. Isot. 52, 669-672, 2000.

6. C. Thiam et al., "Radiopharmaceutical ${ }^{11} \mathrm{C}$ activity measurements by means of the TDCRCerenkov method based on a Geant 4 stochastic modeling”, LSC-2010 Conference, pp. 341-348.

7. S.W. Harmer et al., "Variation in optical constants between photocathodes", Nucl. Instrum. Meth. A 564:439-450, 2006.

8. D. Motta, S. Schönert, "Optical properties of bialkali photocathodes". Nucl. Instrum. Meth. A 539:217-235, 2005.

9. C. Bobin et al., "On the stochastic dependence between photomultipliers in the TDCR method", App. Radiat. Isot., vol. 70, pp. 770-780, 2012. 